\title{
(2) OPEN ACCESS \\ How can patient-held lists of medication enhance patient safety? A mixed-methods study with a focus on user experience
}

\author{
Sara Garfield (D) , ${ }^{1,2,3}$ Dominic Furniss, ${ }^{4}$ Fran Husson, ${ }^{1}$ Mike Etkind, ${ }^{3}$ \\ Marney Williams, ${ }^{1}$ John Norton, ${ }^{2}$ Della Ogunleye, ${ }^{3}$ Barry Jubraj, ${ }^{5,6}$ \\ Hanaa Lakhdari, ${ }^{3}$ Bryony Dean Franklin (1) 1,2,3
}

- Additional material is published online only. To view please visit the journal online (http://dx.doi.org/10.1136/ bmjas-2019-010194).

'Pharmacy department, Imperial College Healthcare NHS Trust, London, UK

${ }^{2}$ Patient Safety Translational Research Centre, Imperial College London, London, UK ${ }^{3}$ Department of Practice and Policy, UCL School of Pharmacy, London, UK

${ }^{4} \mathrm{UCLIC}, \mathrm{UCL}$, London, UK ${ }^{5}$ NIHR CLAHRC, London, UK ${ }^{6}$ Medicines Use \& Safety Division, Specialist Pharmacy Service, London, UK

\section{Correspondence to} Dr Sara Garfield, Imperial College Healthcare NHS Trust, London W2 1NY, UK; sara.garfield@nhs.net

Received 8 August 2019 Revised 16 December 2019 Accepted 22 December 2019 Published Online First

16 January 2020

\section{Check for updates}

(c) Author(s) (or their employer(s)) 2020. Re-use permitted under CC BY-NC. No commercial re-use. See rights and permissions. Published by BMJ.

To cite: Garfield S, Furniss D, Husson F, et al. BMJ Qual Saf 2020:29:764-773.

\begin{abstract}
Background Patients often carry medication lists to mitigate information loss across healthcare settings. We aimed to identify mechanisms by which these lists could be used to support safety, key supporting features, and barriers and facilitators to their use.

Methods We used a mixed-methods design comprising two focus groups with patients and carers, 16 semistructured interviews with healthcare professionals, 60 semistructured interviews with people carrying medication lists, a quantitative features analysis of tools available for patients to record their medicines and usability testing of four tools. Findings were triangulated using thematic analysis. Distributed cognition for teamwork models were used as sensitising concepts. Results We identified a wide range of mechanisms through which carrying medication lists can improve medication safety. These included improving the accuracy of medicines reconciliation, allowing identification of potential drug interactions, facilitating communication about medicines, acting as an aide-mémoire to patients during appointments, allowing patients to check their medicines for errors and reminding patients to take and reorder their medicines. Different tools for recording medicines met different needs. Of 103 tools examined, none met the core needs of all users. A key barrier to use was lack of awareness by patients and carers that healthcare information systems can be fragmented, a key facilitator was encouragement from healthcare professionals.

Conclusion Our findings suggest that patients and healthcare professionals perceive patient-held medication lists to have a wide variety of benefits. Interventions are needed to raise awareness of the potential role of these lists in enhancing patient safety. Such interventions should empower patients and carers to identify a method that suits them best from a range of options and avoid a 'one size fits all' approach.
\end{abstract}

\section{BACKGROUND}

Poor information transfer across healthcare settings has been identified as a major cause of medication errors. ${ }^{12}$ Studies suggest that in the hospital setting, prescribing errors are most common at admission, ${ }^{3}$ largely due to challenges of medication reconciliation. ${ }^{4} 5$ Problems are also common following transfer from hospital into the community ${ }^{67}$ and when attending outpatient appointments. ${ }^{8}$ A survey completed by 113 London general practitioners (GP) to identify priorities for improving medication safety in primary care suggested addressing incomplete medication reconciliation as the highest priority. ${ }^{9}$

Supporting patient (and carer) involvement with their medication is a potential approach to improving information transfer across care settings. Studies have shown that patients who feel in control, empowered and confident have better outcomes. ${ }^{10}$ Supporting greater patient involvement is a fundamental component of 'person-centred care'. ${ }^{11}$ Fylan et al ${ }^{12}{ }^{13}$ also identified patient and carer involvement in medicines management after hospital discharge as an important form of resilience. It is therefore important to consider how to optimise patient and carer involvement in transferring medication-related information across care settings. In England, medication prescribed by a patient's GP is generally listed on their electronic summary care record, which can increasingly be accessed by other healthcare professionals. Some aspects may also be viewable by patients. However, these records do not include over-the-counter or some specialist medication, are sometimes inaccurate and may have limited functionality. Some patients who take medication use various types of medication lists. These may be paper, electronic or a combination of both. Such lists have been included as 
part of a medication reconciliation toolkit. ${ }^{14}$ Previous research ${ }^{15-26}$ has suggested that many patients bring some form of medication list with them to hospital. In these studies, barriers to successfully using such lists included confusion over who was responsible for updating it, lack of understanding as to its purpose, practicalities such as whether it fits into a pocket and lack of space to record potentially important details such as patient preferences for administration. A suggested facilitator was patient-held medication lists being perceived to meet a clear need for potential users.

While such research has identified some barriers and facilitators to use of patient-held medication lists, there has been no formal study of these, the key features of such lists or their role in preventing medication errors. Our aims were therefore to identify how patient-held medication lists are used to support patient safety, key features that support this and barriers and facilitators to their use.

\section{METHODS}

\section{Study design and participants}

We conducted a mixed-methods descriptive study, with the full protocol published elsewhere. ${ }^{27}$ We included patients, carers and healthcare professionals from the Greater London area. Patients were eligible to participate if they had had at least one long-term condition for at least a year, and took at least one prescribed medication.

\section{Definition of patient-held medication list}

We defined a patient-held medication list as any tool that allowed a list of current medications to be edited and carried by patients, regardless of whether or not other functionalities were also available. We included both paper and electronic tools, including printed repeat medication lists, structured paper medication records, medication diaries, apps and websites that could be accessed on a smartphone. We excluded supplies of patients' own drugs, drug-specific tools such as warfarin booklets and patient portals allowing read-only access to healthcare professionals' records.

\section{Theoretical framework}

Communication about medication involves information processing across people, places, tools and artefacts. Distributed cognition is a theoretical framework specifically designed to understand these kinds of sociotechnical systems (see online supplementary appendix 1). ${ }^{28}$ It uses cognitive framing, based on information processing concepts, to explore interactions that are distributed across members of a social group, across internal and external structures and over time. Distributed cognition for teamwork (DiCoT) is a framework that facilitates application of distributed cognition in practice. ${ }^{29}{ }^{30}$ It helps consider different information flows within the system (information flow model), how they are influenced by people (social model) and tools and artefacts (artefact model), how information is processed over physical spaces (physical model) and how it evolves over time (evolutionary model). We used these DiCoT models to inform the development of our topic guides and in our analysis.

\section{Qualitative exploration of the context of patient-held medication lists}

First, we conducted two focus groups. Twenty adult patients/adult carers of adults or children were purposively sampled to include patients and carers, and both users and non-users of patient-held medication lists, to represent a range of genders, ages, ethnicities and localities. Potential participants were asked to complete a screening tool to confirm eligibility and enable purposive sampling. This asked if they carried any form of medication list with them at any time, either on paper or on their smartphone. Participants were recruited from hospitals, patient and carer groups, charities and social media networks. In parallel, we conducted 16 semistructured interviews with healthcare professionals. We used purposive sampling with the aim of creating a maximum variation sample with respect to profession, gender, age, ethnicity, locality and previous experience with patient-held medication lists. We interviewed two GPs, two hospital doctors, two community pharmacists, two hospital pharmacists, two practice nurses, two hospital nurses, two dentists and two opticians. Participants were recruited from healthcare organisations in Greater London, using the authors' personal and professional networks as well as local clinical commissioning groups. Topic guides for focus groups and interviews are included in online supplementary appendix 2 ; all were digitally recorded and professionally transcribed.

Next, we conducted semistructured interviews with 60 people who carried medication lists, including both adults and adult carers of children. We sampled digital and paper-based users, and basic and more extensive users. 'Basic users' were those keeping a list of current regular medications; 'extensive users' were those also recording previous medications, short-term courses of medication, allergies, side effects and/or other information. We used posters, social media and direct approaches in clinical waiting areas to recruit participants. We interviewed participants using a topic guide (see online supplementary appendix 2), took field notes and photographed medication lists with participants' consent.

\section{Quantitative features analysis of existing tools}

We collated a list of desired features based on our empirical data, relevant literature and discussion with key stakeholders. We obtained a list of tools available in the UK for recording medication using a systematic search on the Apple App Store, Google Play, Google, Pinterest, websites of patient charities, plus those 
already known to the research team or identified from our data. The first 45 apps appearing in searches on Apple and Google Play platforms were assessed and those that met our definition of a patient-held medication list included. All paper tools and all tools identified from other sources were also included.

We evaluated usability of four of the tools in more detail: the paper tool and the digital tool that were most frequently reported by our study participants, and the first app to appear on each of the Google Play and Apple App Store. Seven testers (four members of the public taking medicines for long-term conditions, a retired health visitor, an academic pharmacist and a pharmacy student; four female and three male) carried out three usability tasks on each of the four tools. These tasks involved (1) inputting seven medicines, (2) editing medicines, and (3) adding notes about ineffective medicines. No specific training was provided to reflect typical use. Testers were given assistance where needed; any such assistance was documented.

\section{Data analysis}

Transcripts, interview notes and photographs were analysed using NVivo to support coding and analysis. DiCoT models were used as sensitising concepts to explore the data, in which their application was used to inspire insights appropriate to the data rather than being used as a rigid coding framework. ${ }^{31}$ The first author, a qualitative researcher with over 20 years' experience, used a combination of deductive analysis using DiCoT models where relevant, and inductive analysis to add emerging themes. A sample of $20 \%$ of all data were analysed by other researchers on the team, one of whom had extensive experience with DiCoT. As a further reliability check, five lay partners received training in basic qualitative analysis and the DiCoT framework, and then independently coded a sample of interviews, focus groups and photographs, an approach used previously. ${ }^{32}$ Analysis between coders was broadly similar. Any discrepancies were resolved through discussion.

We conducted a descriptive quantitative summary of the tools identified and mapped these against the list of key features. All findings were then triangulated with a focus on patient safety, the features needed to support this, and barriers and facilitators to use of patient-held medication lists.

\section{RESULTS}

The two focus groups with patients/carers were approximately $90 \mathrm{~min}$ duration. In total, 16 participants took part (4 male; 12 female); these comprised seven patients, six carers and three with both roles. Eleven carried medication lists and five did not. Four further patients and carers were recruited but unable to attend on the day. The interviews with 16 healthcare professionals ( 7 male; 9 female) were approximately $30 \mathrm{~min}$ duration. We then conducted
15 interviews with each of the following: basic paper users, extensive paper users, basic digital users and extensive digital users. Their mean age was 53 years (range 18-89). Theoretical saturation was reached.

In total, we assessed 103 tools (57 apps, 42 paperbased tools and 4 websites; online supplementary appendix 3).

\section{Participants' views of the purpose and value of medication lists in enhancing medicines safety}

A wide range of mechanisms were identified through which patient-held medication lists addressed gaps and improved patient safety (table 1 ).

For many participants who carried medication lists and many healthcare professional participants, an important purpose of these was to fill in gaps in information flow between different people to enable an accurate and complete record of medicines to be produced.

To make sure that I give them accurate answers and not partial answers. (Focus group 1 participant)

Participants also had different views on what constituted a complete, accurate list. For example, there were a range of views on whether over-the-counter medicines should be included and who should be responsible for updating the list.

Personally think it should be the clinicians or the medical staff purely because I think if you're asking the patient to change it then let's supposing accidentally they change it to the wrong, you know, their perception of something may be slightly different. (GP1)

It's no good saying a healthcare professional because they could be under the administrations of more than one healthcare professional. Each doctor, nurse or pharmacist that you are dealing with may well only have a partial set of information about you. You're the only person who's got every bit of information. (Focus group 1 participant)

I think it might be a joint thing so... having the entries and going through it with the patient, so maybe the GP probably would be important to assist as well but also patient centred I think is important. (GP2)

It became clear that patient-held medication lists may be needed for information transfer in emergency situations when the patient may not be able to provide the information themselves or where their carer may not be present, and for communication in nonemergency settings. Some healthcare professionals and participants who carried medication lists were of the view that these worked best when accompanied by a verbal conversation.

It's good as well to be able to talk through that information. It's a good way to start the conversation, start building that therapeutic relationship. (Hospital pharmacist 1) 


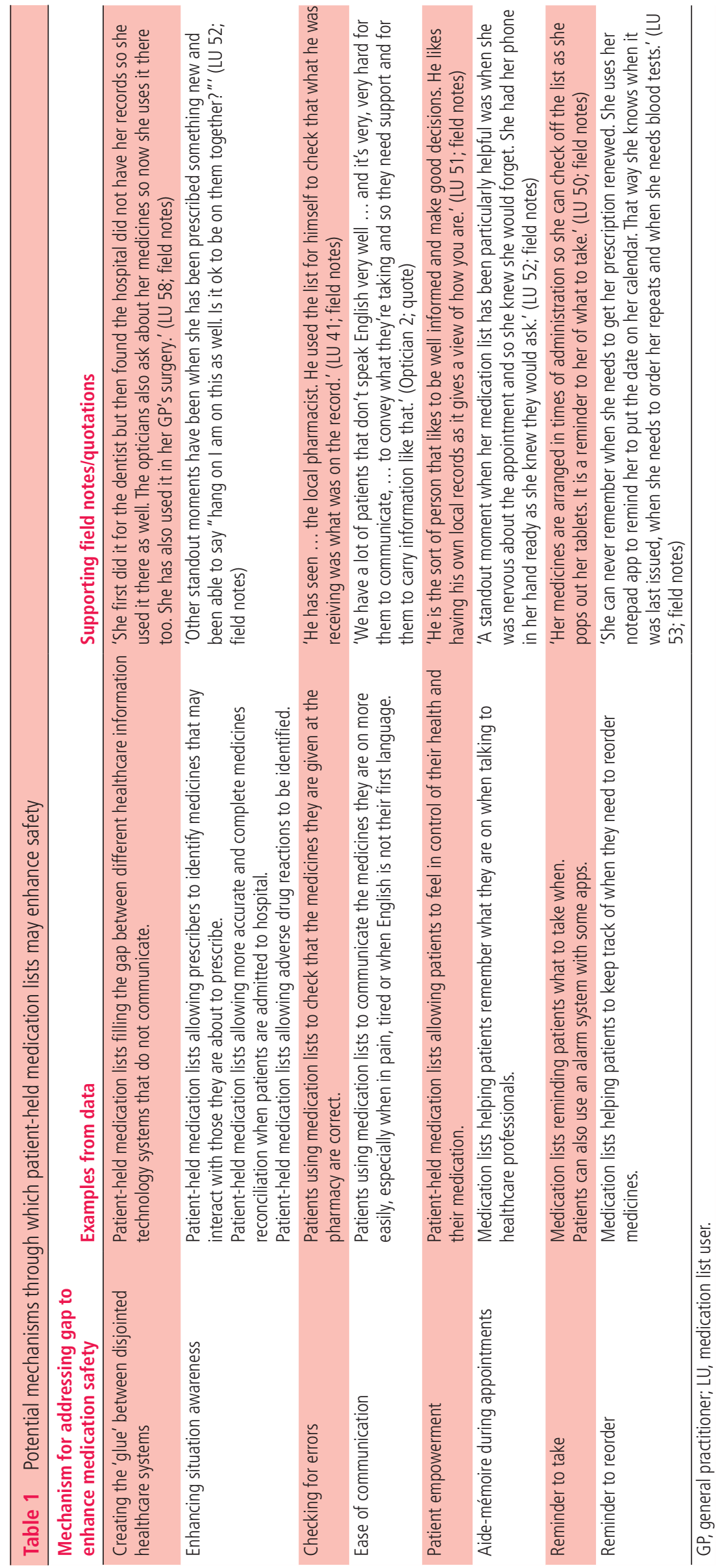


Uses unrelated to information flow described by both those who carried medication lists and healthcare professionals included enabling patient empowerment, assisting with taking and reordering medicines, monitoring healthcare conditions and tracking whether or not medicines were proving effective. These were described by some who carried medication lists as the main reasons for doing so.

It became apparent that different types of medication lists were suitable for different ways of enhancing safety. Some participants reported using more than one type of list for different reasons, such as one for generating reminders to take their medicines and another for emergency access to medicine lists, or one for quick reference in appointments and another to help track their condition over time.

\section{Design of tools for recording medicines}

Having established the roles that medication lists were reported to play in patient safety, we now discuss the features of such lists that optimise safety, the extent to which current tools meet these, and the usability of some of available tools.

\section{Content needed to enhance safety}

Interview data suggested that incorporation of key content (see online supplementary appendix 4) could enhance the role of patient-held medication lists in enhancing patient safety. However, there was some disconnect between the information needs of the majority of healthcare professional participants and the information being kept by most participants who carried medication lists. The majority of healthcare professional participants wanted information about drug names, doses, indications and medicine-related allergies, although some reported that they did not necessarily need all this information. The vast majority of those keeping medication lists recorded the generic name and dose of the medicines that they were taking and allergies if they had any. However, far fewer included indication. On questioning, the majority reported they would be happy to do so but had not thought it necessary as they believed that healthcare professionals would know the purpose of the medicines they were taking.

In addition to this key content, we also identified different features that were important to different users depending on personal preferences and on how they were using the tools to support medication safety. Some used standard tools, whereas others created their own. Participants reported advantages of having a standardised tool to include it becoming part of the established healthcare professionals' workflow, and recognition by all in an emergency. Disadvantages were that patients and carers may not then be able to customise it for their own needs and that standardised tools may result in a document that is healthcare professional rather than patient led.
If there was a specific tool or a booklet a bit like pregnant women have their own notes that they carry around, it's just part of the culture of being pregnant or I think when children are little they have the Red Book [parent-held child health record]. (Hospital doctor 1)

I would have thought people's differing lifestyles as such, it would be very ... you have less chance of success if you tried to make just one single format. (Focus group 1 participant)

Our study suggests that more patients are currently using paper rather than digital medication lists. The vast majority of those carrying lists who we recruited from clinics used paper-based tools; we needed to use a targeted social media recruitment strategy to recruit most of our digital users. In addition, some digital users also used paper tools. Only one healthcare professional participant reported a patient using a digital medication list during a consultation. However, those using digital lists were of the view that digital tools had several advantages over paper. Some had begun using paper and then moved to digital. The advantages most commonly cited were availability (as a smartphone would always be with the user), sustainability (as paper could become worn and ripped) and ease of updating.

Most people have phones on them now don't they? I do think that's a good idea because that could be updated as well so it's always correct. (Focus group 2 participant)

Conversely, several advantages of paper over digital were cited including flexibility, accessibility and that many of the most vulnerable patients may not possess or be comfortable with smartphone technology.

A wide range of paper tools were used. The tool most commonly reported was the repeat (refill) prescription list issued by the patient's GP. Hospital communications were also commonly reported; these included clinic letters, discharge summaries and copies of outpatient prescriptions. Other paper tools included My Medication Passport, ${ }^{17}$ a Parkinson's treatment card, allergy plan, labels from multicompartment compliance aids, a Medic Alert card and a medication administration record chart adapted by a parent from a document produced by a care agency. A large number of patients/parents had created their own paper-based medication lists. These varied greatly, including a list of medicines on a self-adhesive note, a printed list and an entire notebook dedicated to recording medication. A range of features were seen in such tools, including organisation of medicines into different lists and colour coding. Some of those carrying medication lists reported building complexity or moving from a healthcare professional generated tool to their own more tailored list over time.

A range of digital tools were also used. The tool most commonly used was the iPhone 'Health app' which is standard on current iPhones. However, interview data 


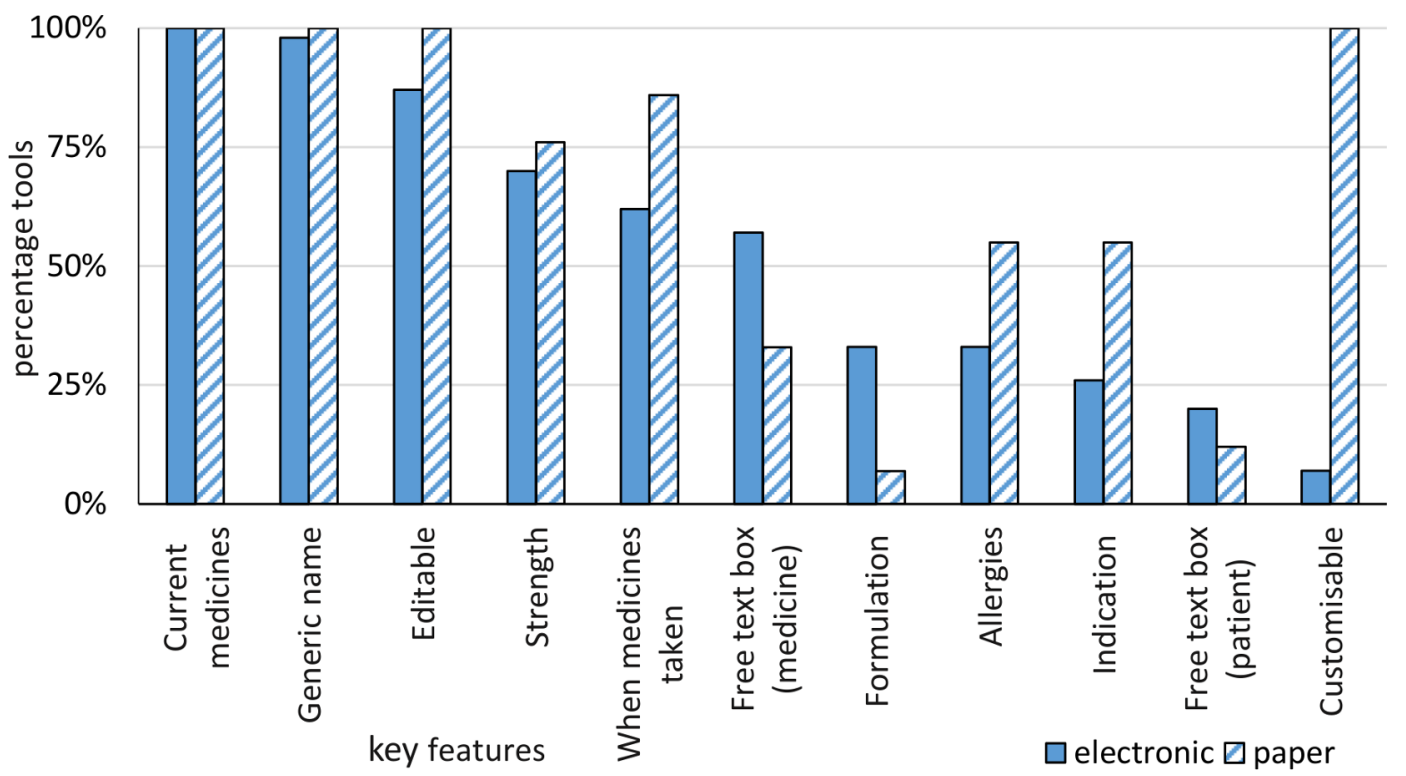

Figure 1 Percentage of electronic and paper tools with core features.

suggested that its potential use for recording medicines was not obvious from the app's front page, which was more geared to use as a fitness tracker. Some participants scanned paper medication lists and kept these on their smartphone. Others created their own digital medication lists using generic features such as smartphone notepad and calendar apps. Others had created a contact called 'medicines' in their contacts list, and then listed their medicines here. Some of these selfcreated digital lists had medicines organised into different categories; none had colour coding.

Mapping of currently available tools against desirable features for enhancing safety

Figures 1-3 show the mapping of tools for recording medicines against the features identified as being important for enhancing safety (see online supplementary appendix 4). Six digital tools (10\%) had a drop-down list of medicines to choose from as well as a free-text option for entering names of medication. No tool had all core features. One digital tool, the Medisafe Pill reminder and medication tracker, had 10 of 11 of the core features but was not customisable. Paper tools were more customisable and more likely to have fields for allergies and indication. All paper tools could be easily shared with others, while just under half of digital tools had the functionality to share the medication list without handing over the phone. All paperbased tools could be accessed in an emergency without a password whereas only two digital tools had this feature. The majority of digital tools had a reminder to take medicines and some also had a reminder feature to order medicines. No paper-based tools had these features.

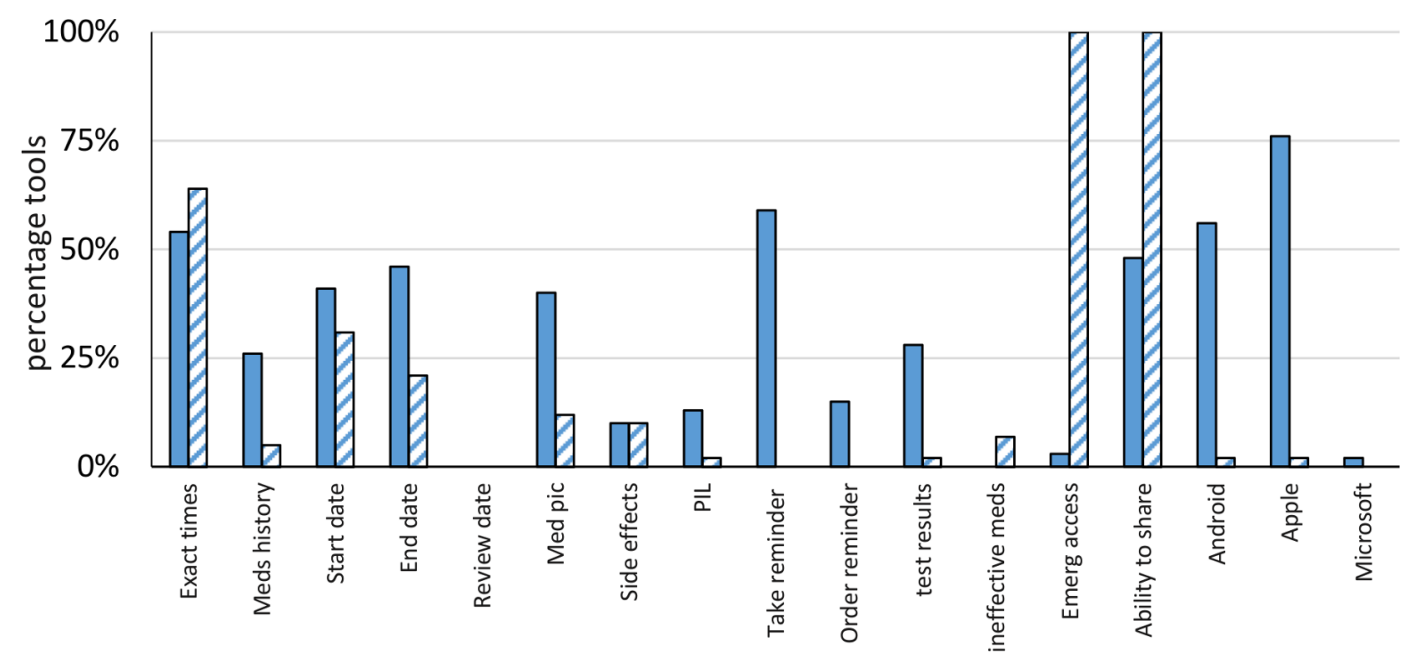

$\square$ electronic $\square$ paper

Figure 2 Percentage of electronic and paper tools with features that may be required by some users. 


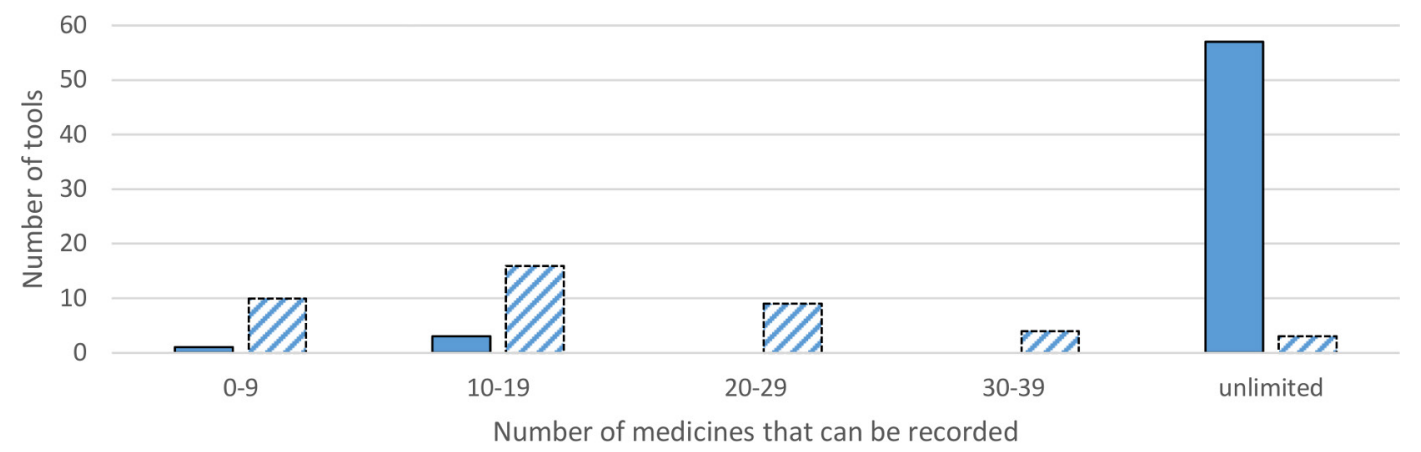

口electronic $\mathbf{a}$ paper

Figure 3 Maximum number of medicines that could be recorded in paper tools.

Usability of tools

Table 2 shows the time taken to complete the usability tasks on each tool.

Usability testing supported the findings that tools were specific to their purpose and mechanism for enhancing safety, that paper was generally preferred to digital and that customisability was an important feature. While several apps whose core purpose was to remind patients to take their medicines also allowed a list of medicines to be produced, not all users felt such lists easily showed all relevant information. Five of the seven users preferred the paper-based tool. However, one stated that she preferred digital tools and completed the tasks on the iPhone Health app quicker than on My Medication Passport. Several testers said that they preferred the iPhone Health app to the other apps as it was simply a free-text box and the experience using continuous typing was more similar to that of paper, rather than having to move between fields. Testers expressed frustration with the MedTracker and Mobile Health apps, perceiving that they would not allow them to insert the information they wanted in the way they wanted, and that they forced them to input information that was not relevant. Three testers did not complete the task involving noting medicines that were ineffective on these apps, saying they could not see how to do it, or not in a way that would be helpful. In contrast, one of the testers stated that the Mobile Health app was their preferred app as it made it clear what information was needed and how to input it.

Table 2 Time to complete usability tasks on the tools

Mean number of minutes taken for seven testers who complete all three Tool tasks (range)

\begin{tabular}{ll}
\hline My Medication Passport (paper based) & $11(6-13)$ \\
\hline iPhone Health app (medication component is free text) & $15(5-27)$ \\
MedTracker (only available on Apple platform) & $27(11-34)$ \\
\hline Mobile Health record (tested on Android platform) & $29(10-60)$ \\
\hline
\end{tabular}

Both testers with a pharmacy background completed the usability testing quicker than the other testers. Several testers were of the view that a drop-down menu for drug names would be helpful.

\section{Barriers}

With the extensive range of tools available and the reported value of these in enhancing safety, we now describe barriers to their use. The main barriers were patients not recognising that healthcare systems were not necessarily connected and that carrying a medication list would be useful, healthcare professionals believing they already had an accurate list and lack of accessibility to the information in an emergency. Barriers identified by smaller numbers of participants were concerns over privacy, carrying something bulky and the challenge of remembering to take their medication lists with them.

Patients assume that we actually have access to their medical record, their default is that we just know and we have everything the GP has. (Community pharmacist 2)

\section{Triggers and facilitators}

Triggers to recognising the value and purpose of patientheld medication lists and starting to use them included the complexity of medicines and being encouraged to do so by healthcare professionals, family or friends. For example, being involved in this study was a trigger for some focus group participants not carrying medication lists to start doing so in the future.

I always assumed the hospitals would know so I think I'll have to get a repeat prescription and just keep that on me. (Focus group 2 participant 1 )

Maybe I should have something about it, I don't know. Yes, it's [this study] made me think twice about it, I must say. (Focus group 2 participant 2)

For some carrying lists of medication, the trigger to start doing so had become less important over time. For example, their condition may have stabilised with 
fewer changes to medicines, and less questioning about medicines at appointments. Or they may have become familiar with their medicines and therefore less reliant on their list as an aide-mémoire or communication tool. One participant said that over time the purpose of his list had changed from use for regular appointments to being carried around in case of emergency.

\section{DISCUSSION}

Our study has identified many mechanisms by which using and carrying patient-held medication lists enhanced patient safety. Such lists were reported to have facilitated and changed the way in which information about medicines was transferred between tools, patients and professionals in different settings.

However, we found a key barrier to use was patients and carers being unaware of the purpose and value of doing so. This was based on the assumption that different healthcare information technology systems communicated with each other. Others carried lists but did not use them in consultations. Facilitators to patients carrying medication lists were identifying a gap in their medication safety and/or being encouraged to do so by healthcare professionals, family or friends. These findings build on those of previous research that has identified that patients need to have a clear purpose for carrying medication lists. ${ }^{18}$ Our study has further identified that patients may not identify this, as they lack knowledge of the level of connection between healthcare systems. Grundy et $a l^{33}$ identified that some medication-related apps, including some we included in our study, share data with third parties. However, privacy was not identified as a major concern for our study participants and none of the apps identified by Grundy $e t a l^{33}$ as sharing data were among those used by our study participants.

While we identified a range of key features that help enhance safety, there was no 'one size fits all' solution. We found great diversity in the tools available for recording medicines and the way in which they were used. None of the available tools met the core needs of all users. While our findings suggest some users would like a standardised tool, many others wanted more flexibility. This is in line with previous research that found My Medication Passport users wanted more space to add information that was relevant to them, for example, to record side effects. ${ }^{18}$ In agreement with Narayan et al, ${ }^{21}$ we found paper solutions to be more popular than digital, although some did prefer digital. Our finding that smartphone ownership can be a barrier is supported by Santo et al, ${ }^{34}$ who found that $39 \%$ of 556 potential participants could not take part in a study of a medication reminder app, as they did not own a suitable smartphone.

\section{Implications for practice and policy}

Our findings suggest that increasing patient and carer awareness of the purpose and value of carrying medication lists would increase medication safety. Findings also suggest that healthcare professionals should not recommend any one particular tool but be aware of several examples of tools they can suggest to patients and carers, and be able to discuss features that may be helpful. While some participants identified advantages of using digital over a paper-based tool, our findings suggest the majority of those carrying medication lists prefer paper-based tools. Healthcare professionals should encourage the use of patient-held medication lists in consultations to facilitate conversations about medicines. Many people who carried information about their medicines only produced these in consultations when they were specifically asked about their medicines.

Tools should be modified to meet all core user requirements identified. Many electronic tools lacked fields for allergies and indication and were not customisable. The majority of electronic tools also had a limit to the number of medicines that could be entered. Relatively few paper or electronic tools had a field for medicine formulation; few had a free-text box to add more information about the user.

\section{Strengths and limitations}

This is the first in-depth study of a wide range of tools for recording medicines, including paper and digital versions. We considered contextual factors relating to the use of such tools, such as their value in enhancing patient safety as well as the tools themselves. Other strengths are our relatively large and varied sample, our mixed-methods methodology and patient and public involvement in data analysis.

A potential limitation is that we interviewed more females than males who carried medication lists. While we had intended the numbers to be equal and used several strategies to increase the number of male participants, we were not able to recruit as many male users. This suggests that more females carry medication lists than males and may therefore reflect current practice rather than being a limitation. The study was limited to one geographical area in the UK at one point in time. Furthermore, our findings suggest the range of tools available is constantly evolving, with three of the digital tools studied no longer available 3 months later. However, a large number of the digital tools evaluated were available internationally and the main findings are likely to be relevant across settings.

\section{CONCLUSION}

Our findings suggest that patient-held medication lists can contribute to the reduction of medication-related risk, particularly in situations of transitions of care and emergency situations. Interventions are needed to raise awareness of the role of patient-held medication lists in enhancing patient safety. Such interventions should empower patients and carers to identify a method that 
suits them best from a range of options, and avoid a 'one size fits all' approach.

Twitter Dominic Furniss@domfurniss and Bryony Dean Franklin@BryonyDF

Contributors SG, FH, BDF and DF conceived and designed the study. SG and HL collected the data. All authors contributed to the analysis of the data. SG wrote the paper. All authors edited the paper and approved the final version.

Funding This work was supported by the Pharmacy Research UK (grant number PRUK-2016-PG2-2-A) and the National Institute for Health Research (NIHR) Imperial Patient Safety Translational Research Centre. This report presents independent research funded by the Pharmacy Research UK (PRUK). Infrastructure support for this research was provided by the NIHR Imperial Biomedical Research Centre. NIHR Clinical Research Network North West London supported the study with recruitment.

Disclaimer The views expressed in this publication are those of the authors and not necessarily those of PRUK, the NHS, the NIHR or the Department of Health and Social Care.

Competing interests None declared.

Patient consent for publication Not required.

Ethics approval This study has been approved by East Midlands-Nottingham 2 Research Ethics Committee (Ref 17/ $\mathrm{EM} / 0477)$.

Provenance and peer review Not commissioned; externally peer reviewed.

Data availability statement Data are available upon reasonable request. Participants did not consent to having their full transcripts publicly available. However, anonymised excerpts are available from the authors who may be contacted on sara. garfield@nhs.net.

Open access This is an open access article distributed in accordance with the Creative Commons Attribution Non Commercial (CC BY-NC 4.0) license, which permits others to distribute, remix, adapt, build upon this work noncommercially, and license their derivative works on different terms, provided the original work is properly cited, appropriate credit is given, any changes made indicated, and the use is noncommercial. See: http://creativecommons.org/licenses/by-nc/4. $0 \%$

\section{ORCID iDs}

Sara Garfield http://orcid.org/0000-0002-9980-0520

Bryony Dean Franklin http://orcid.org/0000-0002-2892-1245

\section{REFERENCES}

1 Mueller SK, Sponsler KC, Kripalani S, et al. Hospital-Based medication reconciliation practices: a systematic review. Arch Intern Med 2012;172:1057-69.

2 Kwan* JL, Lo* L, Sampson M, et al. Medication reconciliation during transitions of care as a patient safety strategy: a systematic review. Ann Intern Med 2013;158:397-403.

3 National Institute for Health and Clinical Excellence. Medicines optimisation: the safe and effective use of medicines to enable the best possible outcomes, 2015. Available: https:// www.nice.org.uk/guidance/ng5 [Accessed 5 Aug 2019].

4 Tully MP, Buchan IE. Prescribing errors during hospital inpatient care: factors influencing identification by pharmacists. Pharm World Sci 2009;31:682-8.

5 Franklin BD, Reynolds M, Shebl NA, et al. Prescribing errors in hospital inpatients: a three-centre study of their prevalence, types and causes. Postgrad Med J 2011;87:739-45.

6 Royal Pharmaceutical Society. Keeping patients safe when they transfer between care providers - getting the medicines right,
2012. Available: http://www.rpharms.com/current-campaignspdfs/rps-transfer-ofcare-final-report.pdf [Accessed 5 Aug 2019].

7 Avery AJ, Ghaleb M, Barber N, et al. The prevalence and nature of prescribing and monitoring errors in English general practice: a retrospective case note review. Br J Gen Pract 2013;63:e543-53.

8 Burnett SJ, Deelchand V, Franklin BD, et al. Missing clinical information in NHS hospital outpatient clinics: prevalence, causes and effects on patient care. BMC Health Serv Res 2011;11:114.

9 Tudor Car L, Papachristou N, Gallagher J, et al. Identification of priorities for improvement of medication safety in primary care: a prioritize study. BMC Fam Pract 2016;17:160.

10 Kings Fund. Supporting people to manage their health. An introduction to patient activation, 2014. Available: https://www.kingsfund.org.uk/sites/default/files/field/field_ publication_file/supporting-people-manage-health-patientactivation-may14.pdf [Accessed 5 Aug 2019].

11 NHs England. The NHS long term plan, 2019. Available: https:/www.longtermplan.nhs.uk/wp-content/uploads/2019/ 01/nhs-long-term-plan.pdf [Accessed 5 Aug 2019].

12 Fylan B, Armitage G, Naylor D, et al. A qualitative study of patient involvement in medicines management after hospital discharge: an under-recognised source of systems resilience. BMJ Qual Saf 2018;27:539-46.

13 Fylan B, Marques I, Ismail H, et al. Gaps, traps, bridges and props: a mixed-methods study of resilience in the medicines management system for patients with heart failure at hospital discharge. BMJ Open 2019;9:e023440.

14 Mueller SK, Kripalani S, Stein J, et al. A toolkit to disseminate best practices in inpatient medication reconciliation: multi-center medication reconciliation quality improvement study (Marquis). Jt Comm J Qual Patient Saf 2013;39:371-3.

15 Koufi V, Malamateniou F, Vassilacopoulos G. An Androidenabled mobile framework for ensuring quality of life through patient-centric care. Stud Health Technol Inform 2012;180:1040-4.

16 Becker S, Kribben A, Meister S, et al. User profiles of a smartphone application to support drug adherence - experiences from the iNephro project. PLoS One 2013;8:e78547.

17 Dharas S, Franklin BD. What Information do Patients Bring into Hospital about Their Medication? [abstract]. London: Royal Pharmaceutical Society, 2014.

18 Barber S, Thakkar K, Marvin V, et al. Evaluation of my medication passport: a patient-completed aide-memoire designed by patients, for patients, to help towards medicines optimisation. BMJ Open 2014;4:e005608.

19 Jubraj B, Blair M. Use of a medication passport in a disabled child seen across many care settings. BMJ Case Rep 2015;2015:bcr2014208033.

20 Becker S, Brandl C, Meister S, et al. Demographic and health related data of users of a mobile application to support drug adherence is associated with usage duration and intensity. PLoS One 2015;10:e116980.

21 Narayan O, Davies S, Tibbins C, et al. Developing a handheld record for patients with cystic fibrosis. Patient Relat Outcome Meas 2015;6:225-8.

22 Lassere MN, Baker S, Parle A, et al. Improving quality of care and long-term health outcomes through continuity of care with the use of an electronic or paper patient-held portable 
health file (communicate): study protocol for a randomized controlled trial. Trials 2015;16:253.

23 Mertens A, Brandl C, Miron-Shatz T, et al. A mobile application improves therapy-adherence rates in elderly patients undergoing rehabilitation: a crossover design study comparing documentation via iPad with paper-based control. Medicine 2016;95:e4446.

24 Saxon LA. Mobile health application solutions. Circulation 2016;9:e002477.

25 Orf K, Tewolde-Berhan L. Implementation of 'My Medication Passport' in the Paediatric Population: Provisional Lessons Gained from Patient and Parent Feedback. Arch Dis Child 2017;102:A91.

26 Waly T, Garfield S, Franklin BD. Pharmacy staff views on the implementation of patient handheld medication tools to improve information transfer: a qualitative study. Saf Health 2018;4:13.

27 Garfield S, Furniss D, Husson F, et al. Use of patient-held information about medication (PHIMed) to support medicines optimisation: protocol for a mixed-methods descriptive study. BMJ Open 2018;8:e021764.
28 Hutchins E. Cognition in the wild. Chicago: MIT Press, 1995.

29 Furniss D, Blandford A. Understanding emergency medical dispatch in terms of distributed cognition: a case study. Ergonomics 2006;49:1174-203.

30 Blandford A, DiCoT FD. A methodology for applying distributed cognition to the design of Teamworking systems. Proceedings of the 12th international conference on Interactive Systems 2005;5:26-38.

31 Bowen GA. Grounded theory and sensitizing concepts. Int J Qual Methods 2006;5:12-23.

32 Garfield S, Jheeta S, Husson F, et al. Lay involvement in the analysis of qualitative data in health services research: a descriptive study. Res Involv Engagem 2016;2:29.

33 Grundy Q, Chiu K, Held F, et al. Data sharing practices of medicines related apps and the mobile ecosystem: traffic, content, and network analysis. BMJ 2019;22:1920.

34 Santo K, Singleton A, Rogers K, et al. Medication reminder applications to improve adherence in coronary heart disease: $\mathrm{a}$ randomised clinical trial. Heart 2019;105:323-9. 